\title{
Efficient Reading Method of a Single Binary Pulse Curve
}

\author{
Si-Yan Wang, Zhao-Xue Chen \\ School of Medical and Food Engineering \\ University of Shanghai for Science and Technology \\ Shanghai, China \\ E-mail: 18238837975@163.com, chenzhaoxue@163.com
}

\begin{abstract}
This paper presents an efficient way to read a single binary pulse curve, which can be used to process paper pulse graphs to achieve the digitized form. For a single binary pulse curve, the single-valued pulse curve data can be obtained by the special designed reading algorithm for a multi-valued curve, which aims at turning multi-valued points to singlevalue points more accurately, at the same time, various kinds of noise can be efficiently removed. Further, the broken points can be connected together based on the linear interpolation method to finish the single pulse reading . Experiments have shown that this method can read a single pulse curve accurately and efficiently.
\end{abstract}

Keywords-binary pulse graphs;single pulse curves; digitized value reading; break points connection-component

\section{INTRODUCTION}

Pulse diagnosis is one of the main diagnostic ways in Chinese medicine[1]. The pulse curve collected by pulse diagnosis instrument can reflect the influence and changing of neuro-hormonal factor under physiological and pathological conditions of the body [2]. Typically,the pulse curve is usually printed on graph paper for the purpose of storage or diagnosis . With the rapid development of science and pulse processing technology, quantities of pulse images have been ccumulated[3] which have put forward many problems such as managing inconvenience, and difficulties to be controlled, stored or analyzed . To overcome these shortcomings to improve efficiency and convenience, it is necessary to research efficient digital extraction technologies for paper-based pulse curves. Under normal circumstances, pulse curve preprocessing technique is firstly used to remove background information and some disturbances or noise, then the pulse curve is detected and extracted to obtain the spacial position of each point of the curve, next the digitized curve is read and stored. Wherein the pulse reading method of binary value curve is a key point.

Pulse curve obtained from scanned images may be inclined or contain grids, patient information and alphabetic marks or other information and thus can be easily polluted by various noise. The single binary pulse obtained by image processing steps such as automatic segmentation, binarization, or waveform position detection usually suffers from problems of discontinuity, noise and uneven thickness. Traditional binary curve reading method is mainly based on smoothing and thinning operations, i.e., a smoothing step is firstly utilized to suppress noise effectively, then the curve similar to the original one is obtained, and the discontinuity points caused by thinning are usually needed to be connected together by a special post-processing step [4]. Generally, smoothing and thinning used for traditional pulse curve reading belongs to morphological methods, during the process of the glitch eliminating and discontinuity points fitting, it is required to track each point multiple times in the region of interest, which is quite time-consuming, and easily led to existence of multi-valued points. Mathematical morphological erosion operation to remove isolated noise is easily led to curve breaking artificially, selecting of structural elements have great influence on the shape of curve by the following broken points connection step[5], which threatens effectiveness and stability of the algorithm.

This paper presents a robust binary pulse curve reading method, which can ensure to obtain a continuous and singlevalued curve. The method eliminates isolated or neighborhood noise points based on the judgment of the distance and the correspondent standard variance ,and gets rid of discontinuity points based on linear interpolation. It can perform single valued reading of the pulse curve without the mathematical morphological operations such as thinning, erosion, smoothing etc.The method implements digitized pulse curve value storage quickly and accurately Firstly, based on the single-valued point extraction algorithm it extracts the value of each point and store them sequentially in a one-dimensional array then performs discontinuity point connection based on linear interpolation algorithm, finally reproduced the curve based on interpolated values. Experimental results show that the algorithm can achieve good reading results for single binary pulse diagnosis curve, which may lay foundation for the realization of a digital pulse curve diagnosis system . It is also of great reference value and significance for common engineering binary curves reading .

\section{THE SINGLE-VALUED POINT READING ALGORITHM FOR BINARY PULSE CURVES WITH MULTI-VALUED PIXEL POINTS}

After steps of rotation correction, grid removing and threshold segmentation, the original pulse image shown in Fig. 1 will be changed into a binary pulse curve image as shown in Fig. 2. Further, a single binary pulse curve can be obtained by certain interactive segmenting or automatic positioning algorithm. In Fig. 2, each single binary pulse diagnosis pulse curve is often not a single-valued one, with defects such as uneven thickness, incoherence and disturbance from various noise etc, which increase the 
difficulty of reading greatly and affect the final accuracy and reliability as well.

The binary value pulse curve extraction algorithm flow chart is shown in Fig. 3.As mentioned above, the basic approach is based on a one-dimensional array. Store the coordinate value of a single pixel on the pulse curve sequentially based on a predetermined reading constraint , then perform discontinuity point connection by a linear interpolation method to attain the goal of value reading.

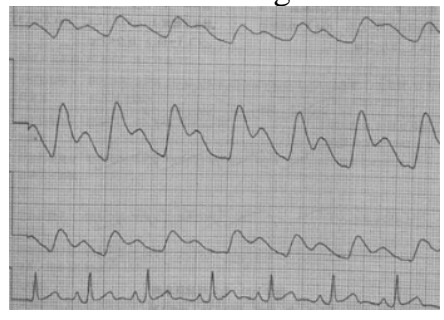

Figure 1. A pulse image.

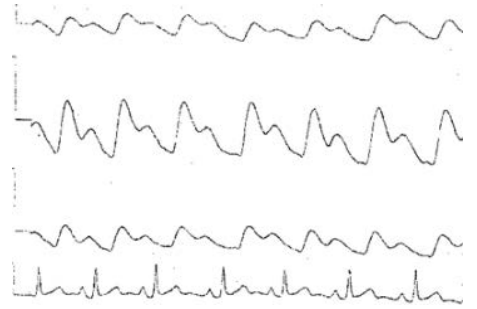

Figure 2. The pulse curve image after image processing

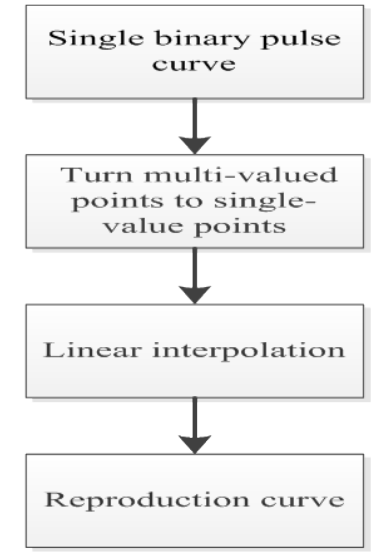

Figure 3 Flow chart of the introduced method.

\section{A. Single Value Reading of the Binary Pulse Curve}

As shown in Fig. 4, generally, there are two sorts of significant influential factors in a single binary pulse curve after image segmentation. Firstly, there are outliers or nonsingle-valued points which both means plurality of values corresponding with the same horizontal coordinate value; Secondly, there are always discontinuities on the curve.

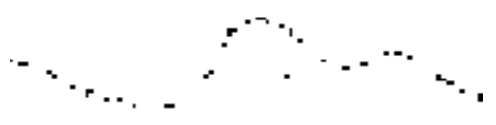

Figure 4 Example for discontinuity points, multi-valued points and utliers

The noise disturbance falls into two kinds, one is the isolated noise, the other is the neighborhood noise. The isolated noise means there is not a single point belongs to the curve segment around it's neighborhood while the neighborhood noise means there are points belongs to curve around its neighborhood. Considering that the binary pulse curve does not have point mutations, and even in the case of thick lines, in which the multi-valued points are usually close to each other, isolated noise points are always outliers far away, the method produced in this paper searches connected line segments vertically at each point along the horizontal axis and calculates the distance between the noise point and those points identified as the curve points at first. If the distance turns out to be less than the predetermined threshold value, the average value will be taken as its "reading" value, otherwise the points will not be "reading" but just be filtered out. All the "reading" values obtained are stored in a one-dimensional array. In the case of multivalued points, such a process suppresses various noises while extracting the information accurately and stably.

In particular, in the implementation process, first of all , the one-dimensional array of elements is initialized to zero, so that if they are discontinuity points they can still remain zero after noise filtering and curve reading .Thus, it can be very easy to distinguish normal values from discontinuity points, which facilitates the following steps.

\section{B. Discontinuity Points Connecting And Curve Filtering}

Unlike the ECG, a pulse curve is relatively smoother, it barely has sudden spikes. Therefore the interpolation method can be used to connect discontinuity points, a simple linear interpolation as below is utilized in this paper to perform discontinuity points connection:

$$
a_{k}=a_{i}+\frac{(k-i)\left(a_{i}-a_{j}\right)}{(i-j)}
$$

Where, $\mathbf{a}_{\mathbf{i}}$ is the starting point of discontinuities in the array $\mathbf{a}_{\mathbf{j}}$ is the ending point of discontinuities in the array. $i$, $\mathrm{j}, \mathrm{k}$ is subscripts of the corresponding element. $\mathbf{a}_{\mathbf{k}}$ is the value used to fill one-dimensional array.

In detail, discontinuity points in the binary curve (as described above, which is initialized as a value of zero in the array), can be recognized at first and are then filled with corresponding interpolation value calculated according to 
(1). Obviously, the interpolation process ensures the the continuity of the curve.

In addition, the median filter can filter grain noises effectively while protect the image details at the same time
[6], further median filter can remove the rest of noise and make the curve smoother for improving the reading accuracy.

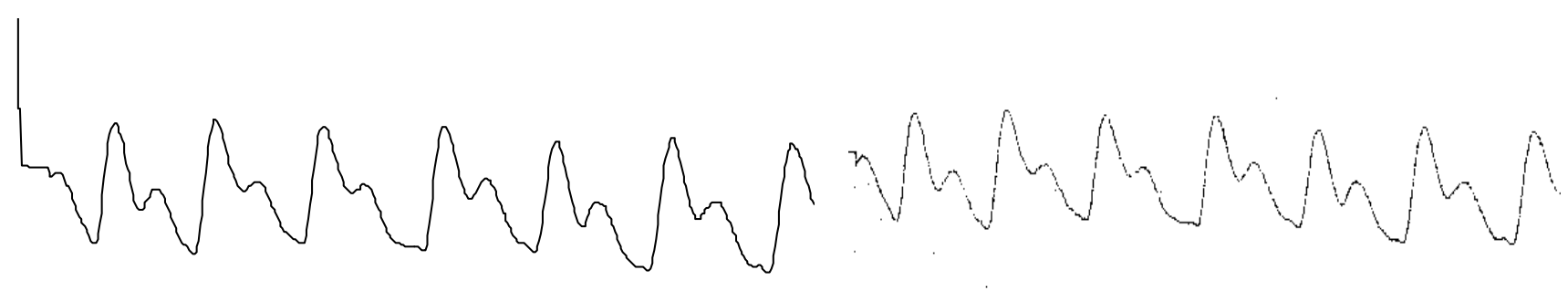

(a)

(b)

Figure 5. An example of original binary pulse curve and the corresponding reproduced curve (a) the original binary pulse curve (b) reproduction of the pulse curve

\section{EXPERIMENTAL RESULTS AND CONCLUSIONS}

The binary pulse curve after steps of rotation correction, grid removing and threshold segmentation, becomes a digitized binary curve. The one-dimensional array with corresponding coordinates is used to reproduce the pulse curve.

To validate the effectiveness of the method proposed, experiments are conducted as shown in Fig 4. To further verify

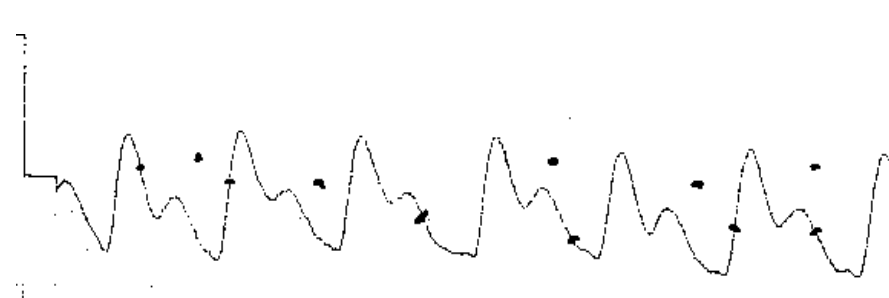

(a) the robust of the proposed method, a simulation experiment is also performed. Among them, the binary pulse curve have been mixed with a large number of different types of noise, which causes the curve have a lot of uneven thickness with some random discontinuities. They are shown in Fig. 5-7.

Figure 6 An example of the curve with different types of noises (a) The original curve (b) The reproduced curve

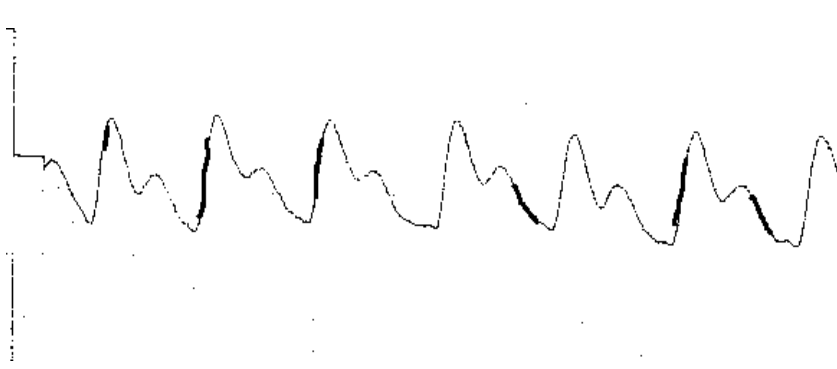

(a)

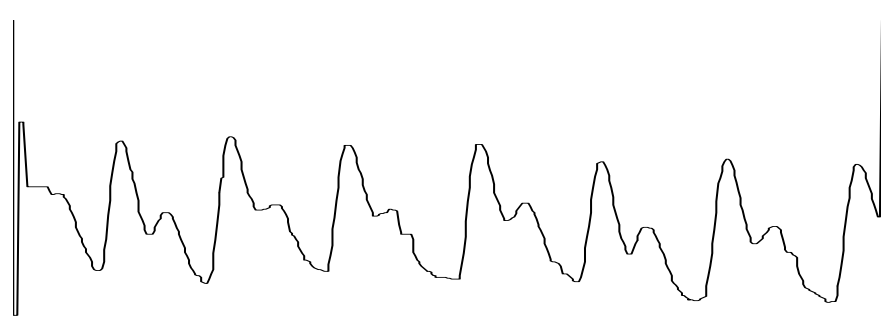

(b)

Figure 7 An example of the curve with uneven thickness (a) The original curve (b) The reproduced curve 


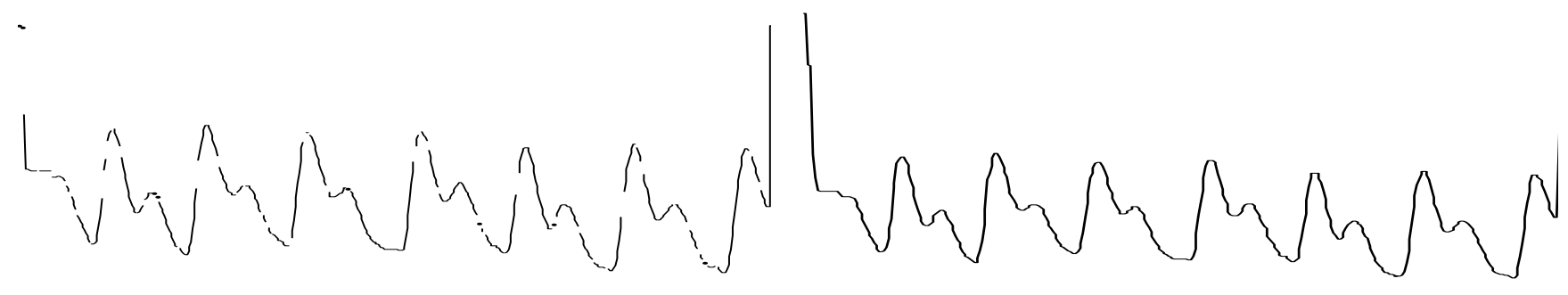

(a)

(b)

Figure 8 An example of the curve with a large number of discontinuities (a) The original curve (b) The reproduced curve.

The simulation results show that the proposed method can achieve accurate reading in the circumstances of a number of different types of mixed noise, a lot of uneven thickness and a large number of discontinuity points. This proves that the method can effectively eliminate different types of noise and connect the discontinuity points with good integrity, stability and accuracy. The method eliminates noise point based on distance, obtains singlevalued curve based on the average of connected components, and implement connection of discontinuity points based on linear interpolation and finally realizes curve reading completely and effectively without thinning, erosion, smooth and other mathematical morphological complex operations, which improves the extracting efficiency of the curve information greatly and lays foundation for the pulse diagnosis curve digital analysis system developing. With broad applicability, the basic principles and ideas can also be extended to other engineering curve reading process.

\section{ACKNOWLEDGMENT}

This work is sponsored by Natural Science Foundation of Shanghai.

\section{REFERENCES}

[1] C Han-Lin, A W H Yang, S Pittayachawan,and N Wickramasinghe. "An analysis on the utilisation of health information technology to support clinical operation of Chinese medicine” Maximizing healthcare delivery and management through technology integration. pp.113-132,. March 2016, doi: 10.4018/978-1-4666-9446-0.ch008

[2] Zhu Qingwen. Research on partable diagnosis and treatment equipments of pulse diagnosis and glossoscopy. Diss.Beijing University of Chinese Medicine , 2007.

[3] Liu, Guang, and H. Sun. "Analysis on Data Pre-processing of TCM Data Mining." Journal of Liaoning University of Traditional Chinese Medicine, vol.14, May. 2012, pp.147-148.

[4] Nonaka, Osamu, and Y. Wani. "IMAGING DEVICE AND IMAGING DEVICE CONTROL METHOD." US, US 20100208107 A1. 2010.

[5] WU Xiao-Ming, XD Lin, H Yuan, RU Chang-Qu . "An automatic method for searching the medical curve in the plotting paper." Journal of Jinan University, vol. 21, pp. 58-60, Feb 2000

[6] Xiao, Xin. "A Coding and Sorting Order Algorithm for Medium Filter."Journal of Computer Aided Design \& Computer Graphics. vol.16, Sep. 2004, pp. 1256-1259. 